\title{
Ricci Lower Bound for Kähler-Ricci Flow
}

\author{
Zhou Zhang \\ Univeristy of Sydney
}

\begin{abstract}
Ricci lower bound along Kähler-Ricci flow with singularity over closed manifold.
\end{abstract}

\section{Introduction and Set-up}

In this note, we study a topic regarding the behaviour of Ricci curvature for Kähler-Ricci flows over closed Kähler manifolds. This relates to the general brief (or conjecture) that Ricci curvature would have uniform lower bound towards (most) finite or infinite time singularities. A typical result is as follows, which is a more English version of Theorem 2.1

Theorem 1.1. For any Kähler-Ricci flow with finite time singularity, if the global volume is not going to zero towards the time of singularity, then the Ricci curvature can not have a uniform lower bound.

Kähler-Ricci flow is nothing but Ricci flow with initial metric being Kähler. For this Kähler condition of the initial metric, the closed smooth manifold, $X$, would be given a complex structure, which is fixed for the consideration. So we still call this complex manifold by $X$. The smooth flow metric would always be Kähler with respect to $X$, as first observed by $\mathrm{R}$. Hamilton. We consider $\operatorname{dim}_{\mathbb{C}} X=n \geqslant 2$.

Thus the standard form of Kähler-Ricci flow, a directly transformation from Ricci flow to a metric form flow, is as follows over $X \times[0, S)$ (for some $S \in(0, \infty]$ )

$$
\frac{\partial \omega(s)}{\partial s}=-2 \operatorname{Ric}(\omega(s)), \quad \omega(0)=\omega_{0},
$$

where $\omega_{0}$ is the metric form for the initial Kähler metric. The key advantage in our study of this flow, comparing with many earlier works, is that we no longer force cohomology restriction to $\left[\omega_{0}\right]$ when setting up this flow (and the equivalent scalar metric potential flow appearing shortly). This allows more applications for this kind of geometric flow techniques and more importantly, makes it possible to analyze degenerate situation. This idea first appeared in [8] and has been rigorized and generalized in the works of [7, [4] and continuations.

We often perform the following time-metric scaling for the above evolution equation,

$$
\omega(s)=e^{t} \widetilde{\omega}_{t}, \quad s=\frac{e^{t}-1}{2},
$$

and arrive at an equivalent version of Kähler-Ricci flow over $X \times[0, T)$ (for $T=\log (1+2 S) \in(0, \infty])$,

$$
\frac{\partial \widetilde{\omega}_{t}}{\partial t}=-\operatorname{Ric}\left(\widetilde{\omega}_{t}\right)-\widetilde{\omega}_{t}, \quad \widetilde{\omega}_{0}=\omega_{0}
$$


which is somewhat more convenient as explained in the following.

To begin with, let's point out that the time scaling makes sure that these two flows would both exist up to some finite times (with finite time singularity) or exist forever. In the finite time singularity case, the metric scaling is by uniformly control positive constants, and so one can say the equivalence is stronger. When they both exist forever, the metric scaling might have significant impact on the flow metric. For example, in the case of $c_{1}(X)=0$ as studied in [1, $\omega(s)$ converges at time infinity to the unqiue Ricci-flat metric in the Kähler class $\left[\omega_{0}\right]$ with the lower $C Y$ standing for the more popular name as Calabi-Yau metric, while $\left(X, \widetilde{\omega}_{t}\right)$ shrinks to a metric point at time infinity. Meanwhile, in other cases, $\omega(s)$ has volume tending to infinty while $\widetilde{\omega}_{t}$ has uniformly controlled volume. In principle, (1.2) always has the cohomology information $\left[\widetilde{\omega}_{t}\right]$ under uniform control, as is clear in following discussion.

For the rest of this note, we focus on (1.2).

Let's fix the convention that [Ric] $=c_{1}(X)$, and then one can reduce (1.2) to an ODE in the cohomology space $H^{1,1}(X, \mathbb{R}):=H^{2}(X ; \mathbb{R}) \cap H^{1,1}(X ; \mathbb{C})$. It's easy to solve it and we end up with

$$
\left[\widetilde{\omega}_{t}\right]=-c_{1}(X)+e^{-t}\left(\left[\omega_{0}\right]+c_{1}(X)\right)
$$

which stands for an interval in this vector space with two endpoints being $\left[\omega_{0}\right]$ for $t=0$ and $-c_{1}(X)$ formally for $t=\infty$. It's easy to see that for (1.1), $[\omega(s)]$ would evolve linearly in general, which might be a simpler function but the control is not as uniform.

However, for either one, the optimal existence result in $[7$ tells that the flow metric exists as long as the class from the above consideration stays inside the open cone, consisting of all Kähler classes (called Kähler cone). In this note, we use $\mathrm{KC}(X)$ to denote the Kähler cone of $X$, and then we call its closure (in the finite dimensional vector space $\left.H^{1,1}(X, \mathbb{R})\right), \overline{\mathrm{KC}(X)}$, is sometime called the numerically effective cone, using a terminology borrowed from Agebraic Geometry. Now the optimal existence result simply says

$$
T=\sup \left\{t \mid-c_{1}(X)+e^{-t}\left(\left[\omega_{0}\right]+c_{1}(X)\right) \in \mathrm{KC}(X)\right\}
$$

is the largest time to consider classic solution for (1.2). This would be our definition for $T$ for the rest of this work, which takes value in $(0, \infty]$. Also from the result in [7, we've already shown that singularity only happens when the class hits the boundary of this cone, at either finite or infinite time. In other words, $\left[\omega_{T}\right] \in \overline{\mathrm{KC}(X)} \backslash \mathrm{KC}(X)$. Of course, now the interesting and in general hard question is to see how the non-Kähler feature of this class at the boundary of $\mathrm{KC}(X)$ and the behaviour of the Kähler-Ricci flow would interact with each other.

The study of this topic, as well as for many other things regarding KählerRicci flow, usually makes of the scalar version of the Kähler-Ricci flow. For (1.2), we define the following background form,

$$
\omega_{t}=-\operatorname{Ric}\left(\omega_{0}\right)+e^{-t}\left(\omega_{0}+\operatorname{Ric}\left(\omega_{0}\right)\right)
$$

compatible with the notation $\omega_{0}$. The whole point is that $\left[\omega_{t}\right]=\left[\widetilde{\omega}_{t}\right]$, and so $\widetilde{\omega}_{t}=\omega_{t}+\sqrt{-1} \partial \bar{\partial} u$. It's not so hard to prove the following scalar evolution 
equation for metric potential $u$ over $X \times[0, T)$ is equivalent to (1.2),

$$
\frac{\partial u}{\partial t}=\log \frac{\widetilde{\omega}_{t}^{n}}{\omega_{0}^{n}}-u=\log \frac{\left(\omega_{t}+\sqrt{-1} \partial \bar{\partial} u\right)^{n}}{\omega_{0}^{n}}-u, \quad u(\cdot, 0)=0 .
$$

This evolution equation can be reformulated as

$$
\left(\omega_{t}+\sqrt{-1} \partial \bar{\partial} u\right)^{n}=e^{\frac{\partial u}{\partial t}+u} \omega_{0}^{n},
$$

which is why sometimes we also call it the complex Monge-Ampère equation type of Kähler-Ricci flow.

Now let's also clarify that the uniform Ricci lower bound mentioned at the beginning. It means that there exists some constant $C$ such that

$$
\operatorname{Ric}\left(\widetilde{\omega}_{t}\right) \geqslant-C \widetilde{\omega}_{t}
$$

uniformly for $t \in[0, T)$. In general, Ricci curvature being bounded from below provides some control on the metric and topology of the underlying manifold. So such a control is certainly favourable as an assumption and interesting as a result in the study of Ricci flow (see in $[9$ for examples of such assumptions).

Note: in the following, $C$ always stands for a (positive) constant, which might be different at places.

\section{Finite Time Singularity}

In this section, we consider the case of $T<\infty$. Then clearly, $\left[\omega_{T}\right] \in \overline{\mathrm{KC}(X)} \backslash$ $\mathrm{KC}(X)$. The following is the main result.

Theorem 2.1. Consider (1.2) with finite time singularity, i.e., $T<\infty$. If $\left[\omega_{T}\right]^{n}>0$, then the Ricci curvature can NOT have a uniform lower bound, i.e., there is $N O$ constant $D>0$ such that $\operatorname{Ric}\left(\widetilde{\omega}_{t}\right) \geqslant-D \widetilde{\omega}_{t}$ uniformly for $t \in[0, T)$.

The proof is a combination of techniques from [11] and [10. We begin with some general situation and finally specify to the case of the above theorem to prove it.

The following is observed earlier as in Remark 2.3 of [10. It's clear that $\left[\omega_{t}\right]^{n}=\left[\widetilde{\omega}_{t}\right]^{n}>0$ for $t \in[0, T)$, and we also have $[\widetilde{\omega}]^{n}=\left[\omega_{t}\right]^{n} \rightarrow\left[\omega_{T}\right]^{n}$ as $t \rightarrow T$. So $\left[\omega_{T}\right]^{n} \geqslant 0$. In exactly the same manner, we see $\left[\omega_{T}\right]^{n-k} \cdot\left[\omega_{0}\right]^{k} \geqslant 0$ for $k=1, \cdots, n-1$. Now rewrite $\omega_{t}$ as follows,

$$
\omega_{t}=\left(\frac{1-e^{-t}}{1-e^{-T}}\right) \omega_{T}+\left(\frac{e^{-t}-e^{-T}}{1-e^{-T}}\right) \omega_{0}
$$

and it is then obvious that for $t \in[0, T]$,

$$
\left[\omega_{t}\right]^{n} \sim(T-t)^{K}
$$

where $K$ is defined as follows,

$$
n \geqslant K:=\min \left\{k \in\{0,1,2, \cdots, n\} \mid\left[\omega_{T}\right]^{n-k} \cdot\left[\omega_{0}\right]^{k}>0\right\},
$$


which is well-defined since $\left[\omega_{0}\right]^{n}>0$. Here $A \sim B$ for $A$ and $B$ both nonnegative means $\frac{1}{C} B \leqslant A \leqslant C B$ for some positive constant $C$.

Note: when $\left[\omega_{T}\right]^{n}>0, K=0$ and $\frac{1}{C} \leqslant\left[\omega_{t}\right]^{n} \leqslant C$ for constant $C>0$.

As in Subsection 2.3 of [10, after assuming $\operatorname{Ric}\left(\widetilde{\omega}_{t}\right) \geqslant-C \widetilde{\omega}_{t}$ for some constant $C>0$, plugging it into (1.2) gives $\frac{\partial \widetilde{\omega}_{t}}{\partial t} \leqslant C \widetilde{\omega}$. Then noticing $T<\infty$, we arrive at $\widetilde{\omega}_{t} \leqslant C \omega_{0}$ and so $\operatorname{Ric}\left(\widetilde{\omega}_{t}\right) \geqslant-C \omega_{0}$.

The equivalent equations (1.2) and (1.3) give

$$
\operatorname{Ric}\left(\widetilde{\omega}_{t}\right)=-\frac{\partial \widetilde{\omega}_{t}}{\partial t}-\widetilde{\omega}_{t}=\operatorname{Ric}\left(\omega_{0}\right)-\sqrt{-1} \partial \bar{\partial}\left(\frac{\partial u}{\partial t}+u\right),
$$

and so one arrives at

$$
C \omega_{0}+\sqrt{-1} \partial \bar{\partial}\left(-\frac{\partial u}{\partial t}-u\right) \geqslant 0 .
$$

Thus we can apply the classic result in [6] and have a constant $\alpha>0$ depending only on $\left(X, \omega_{0}\right)$ such that for $t \in[0, T)$,

$$
\int_{X} e^{\alpha\left(\sup _{X}\left(-\frac{\partial u}{\partial t}-u\right)+\left(\frac{\partial u}{\partial t}+u\right)\right)} \omega_{0}^{n} \leqslant C .
$$

Of course, we could make sure $\alpha \leqslant 1$. This gives

$$
\inf _{X}\left(\frac{\partial u}{\partial t}+u\right) \geqslant \frac{1}{\alpha} \log \left(\frac{1}{C} \int_{X} e^{\alpha\left(\frac{\partial u}{\partial t}+u\right)} \omega_{0}^{n}\right) .
$$

As summarized in [10], we have $\frac{\partial u}{\partial t}+u \leqslant C$, and so

$$
\begin{aligned}
\int_{X} e^{\alpha\left(\frac{\partial u}{\partial t}+u\right)} \omega_{0}^{n} & =e^{\alpha C} \int_{X} e^{\alpha\left(\frac{\partial u}{\partial t}+u-C\right)} \omega_{0}^{n} \\
& \geqslant e^{\alpha C} \int_{X} e^{\frac{\partial u}{\partial t}+u-C} \omega_{0}^{n} \\
& \geqslant C \int_{X} e^{\frac{\partial u}{\partial t}+u} \omega_{0}^{n} \\
& =C\left[\widetilde{\omega}_{t}\right]^{n}=C\left[\omega_{t}\right]^{n} \geqslant C(T-t)^{K}
\end{aligned}
$$

where $\alpha \leqslant 1$ is applied for the second step. So we conclude that for $t \in[0, T)$,

$$
\inf _{X}\left(\frac{\partial u}{\partial t}+u\right) \geqslant-C+\frac{K}{\alpha} \log (T-t)
$$

and so

$$
\frac{\partial u}{\partial t}+u \geqslant-C+\frac{K}{\alpha} \log (T-t)
$$

for $\alpha \in(0,1]$ depending only on $\left(X, \omega_{0}\right)$. Directly applying Maximum Principle to (1.3), we have $u \leqslant C$ and so

$$
\frac{\partial u}{\partial t} \geqslant-C+\frac{K}{\alpha} \log (T-t) .
$$

So in a way slightly different from that in $[10$, we have $u \geqslant-C$.

The above estimate provides a pointwise lower bound of the volume form, $\widetilde{\omega}_{t}^{n}=e^{\frac{\partial u}{\partial t}+u} \omega_{0}^{n}$. Combining with the metric upper bound, we arrive at the following proposition. 
Proposition 2.2. Consider (1.2) with singularity at some finite time $T$. If $\operatorname{Ric}\left(\widetilde{\omega}_{t}\right) \geqslant-D \widetilde{\omega}_{t}$ for some constant $D>0$ and $t \in[0, T)$, then

$$
(T-t)^{\beta} \omega_{0} \leqslant \widetilde{\omega}_{t} \leqslant C \omega_{0}
$$

for positive constants $\beta$ and $C$ depending on $X, \omega_{0}, T$ and $D$.

Now we restrict to the case of Theorem 2.1. In this case, $K=0$ and so the above lower bound for $\frac{\partial u}{\partial t}$, (2.1) is uniform. Hence, the metric control in Proposition 2.2 is also uniform. The argument in 11 can be used to draw contradiction with the existence of finite time singularity at $T$. Theorem 2.1 is thus proven.

Remark 2.3. This theorem indicates that for the problem in [10] on general weak limit, when Ricci curvature has uniform lower bound, the discussion there is actually only for the global volume collapsed case. This again stresses the point that the discussion of collapsed case is the core for the topic of weak limit in general.

It's worth pointing out that there are numerous examples satisfying the assumption of this theorem. For instance, we have the case discussed in [5]. In fact, such manifold of $X$ belongs to the class of so-called manifolds of general type, indicating "majority".

The problem on finite time singularity has been studied extensively since R. Hamilton's original work [2]. The Ricci lower bound assumption and $N$. Sesum's result on the blow-up of Ricci curvature for finite time singularity of Ricci flows over closed manfolds in [3] automatically gives the blow-up of the scalar curvature, which is conjectured in general and proven for Kähler case in [11]. Our theorem here actually shows that the situation has to be more complicated, at least in the global volume non-collapsed case.

\section{Infinite Time Singularity}

Now we consider the infinite time singularity case, i.e., $T=\infty$ and $\left[-\operatorname{Ric}\left(\omega_{0}\right)\right]=$ $-c_{1}(X) \in \overline{\mathrm{KC}(X)} \backslash \mathrm{KC}(X)$. When $X$ is projective, it is then a minimal manifold. Again we assume $\operatorname{Ric}\left(\widetilde{\omega}_{t}\right) \geqslant-D \widetilde{\omega}_{t}$, which clearly gets weaker as $D$ gets larger. Our discussion below is separated into cases for increasing $D$ value, with the conclusion getting weaker.

- $D<1$

In this case, (1.2) gives $\frac{\partial \widetilde{\omega}_{t}}{\partial t} \leqslant(D-1) \widetilde{\omega}_{t}$, and so $\widetilde{\omega}_{t} \leqslant e^{(D-1) t} \omega_{0}$.

So clearly $-c_{1}(X)=\left[-\operatorname{Ric}\left(\omega_{0}\right)\right]=0$, and the result in [1] can be scaled to provide a very satisfying description of the flow metric as follows. Using the notations in Section 1, $\omega(s)=e^{t} \widetilde{\omega}_{t}$ converges exponentially fast (for example, Section 9.3 in [13]) to the Ricci-flat Kähler metric $\omega_{C Y}$, where this exponentially fast convergence is with respect to the parameter $s=$ $\frac{e^{t}-1}{2}$. So as smooth forms, $\operatorname{Ric}\left(\widetilde{\omega}_{t}\right)$ is $e^{-s}$-small while $\widetilde{\omega}_{t}$ is $e^{-t}$-positive, and so the above Ricci lower bound is obviously true for large time.

- $D=1$

In this case, (1.2) gives $\frac{\partial \widetilde{\omega}_{t}}{\partial t} \leqslant 0$, and so $\widetilde{\omega}_{t} \leqslant \omega_{0}$. 
$\operatorname{Ric}\left(\widetilde{\omega}_{t}\right)+\widetilde{\omega}_{t} \geqslant 0$ also tells us that the corresponding cohomology class

$$
-c_{1}(X)+\left(-c_{1}(X)+e^{-t}\left(\left[\omega_{0}\right]+c_{1}(X)\right)\right)=e^{-t}\left(\left[\omega_{0}\right]+c_{1}(X)\right) \in \overline{\mathrm{KC}}(X),
$$

and so $\left[\omega_{0}\right]+c_{1}(X) \in \overline{\mathrm{KC}}(X)$, providing a topological restriction.

The above uniform metric upper bound allows most of the discussion in Section 2 to be carried through. Together with $\operatorname{Ric}\left(\widetilde{\omega}_{t}\right) \geqslant-\widetilde{\omega} \geqslant-\omega_{0}$,

$$
\operatorname{Ric}\left(\widetilde{\omega}_{t}\right)=-\frac{\partial \widetilde{\omega}_{t}}{\partial t}-\widetilde{\omega}_{t}=\operatorname{Ric}\left(\omega_{0}\right)-\sqrt{-1} \partial \bar{\partial}\left(\frac{\partial u}{\partial t}+u\right),
$$

will give us

$$
C \omega_{0}+\sqrt{-1} \partial \bar{\partial}\left(-\frac{\partial u}{\partial t}-u\right) \geqslant 0
$$

Again apply the classic result in [6] to get constant $\alpha>0$ depending only on $\left(X, \omega_{0}\right)$ such that for $t \in[0, \infty)$,

$$
\int_{X} e^{\alpha\left(\sup _{X}\left(-\frac{\partial u}{\partial t}-u\right)+\left(\frac{\partial u}{\partial t}+u\right)\right)} \omega_{0}^{n} \leqslant C .
$$

Of course, we could make sure $\alpha \leqslant 1$. This gives

$$
\inf _{X}\left(\frac{\partial u}{\partial t}+u\right) \geqslant \frac{1}{\alpha} \log \left(\frac{1}{C} \int_{X} e^{\alpha\left(\frac{\partial u}{\partial t}+u\right)} \omega_{0}^{n}\right) .
$$

As summarized in [10, we still have $\frac{\partial u}{\partial t} \leqslant C$ and $u \leqslant C$, and so in the same way as in Section 2, we arrive at

$$
\int_{X} e^{\alpha\left(\frac{\partial u}{\partial t}+u\right)} \omega_{0}^{n} \geqslant C\left[\omega_{t}\right]^{n}
$$

Repeating the same discussion at the beginning of Section 2, we have $\left[-\operatorname{Ric}\left(\omega_{0}\right)\right]^{n-k} \cdot\left[\omega_{0}\right]^{k} \geqslant 0$ for $k \in\{0,1, \cdots, n\}$, where $-\operatorname{Ric}\left(\omega_{0}\right)$ can be viewed as $\omega_{T}$ for $T=\infty$. Furthermore, $\left[\omega_{t}\right]^{n} \sim e^{-K t}$ with

$$
n \geqslant K:=\min \left\{k \in\{0,1,2, \cdots, n\} \mid\left[-\operatorname{Ric}\left(\omega_{0}\right)\right]^{n-k} \cdot\left[\omega_{0}\right]^{k}>0\right\},
$$

which is well-defined since $\left[\omega_{0}\right]^{n}>0$. So we conclude that for $t \in[0, \infty)$,

$$
\inf _{X}\left(\frac{\partial u}{\partial t}+u\right) \geqslant-\frac{K}{\alpha} t-C,
$$

and so

$$
\frac{\partial u}{\partial t}+u \geqslant-\frac{K}{\alpha} t-C
$$

for $\alpha \in(0,1]$ depending only on $\left(X, \omega_{0}\right)$. This provides a pointwise lower bound of the volume form, $\widetilde{\omega}_{t}^{n}=e^{\frac{\partial u}{\partial t}+u} \omega_{0}^{n}$. Combining with the metric upper bound, we arrive at the following proposition.

Proposition 3.1. Consider (1.2) with the solution existing forever but having infinite time singularitiy. If $\operatorname{Ric}\left(\widetilde{\omega}_{t}\right) \geqslant-\widetilde{\omega}_{t}$ for $t \in[0, \infty)$, then $\left[\omega_{0}\right]+c_{1}(X) \in \overline{\mathrm{KC}}(X)$ and

$$
e^{-\beta t} \omega_{0} \leqslant \widetilde{\omega}_{t} \leqslant \omega_{0}
$$

for some positive constant $\beta$ depending on $\left(X, \omega_{0}\right)$. 
If we further assume $K=0$, i.e., $\left[-\operatorname{Ric}\left(\omega_{0}\right)\right]^{n}>0$, then it's the global volume non-collapsed case and the metric bound from the above proposition is uniform. As in [1], this implies $\left[-\operatorname{Ric}\left(\omega_{0}\right)\right]=-c_{1}(X) \in \operatorname{KC}(X)$, which contradicts the infinite time sinuglarity assumption which indicates $\left[-\operatorname{Ric}\left(\omega_{0}\right)\right] \in \overline{\mathrm{KC}(X)} \backslash \mathrm{KC}(X)$. Let's summarize it in the following corollary, which is similar to Theorem 2.1 but not as neat.

Corollary 3.2. Consider (1.2) with the solution exists forever but having infinite time singularity. If $\operatorname{Ric}\left(\widetilde{\omega}_{t}\right) \geqslant-\widetilde{\omega}_{t}$ for $t \in[0, \infty)$, then $c_{1}(X)^{n}=0$.

- $D>1$

This is the general case. We can only have $\widetilde{\omega}_{t} \leqslant e^{C t} \omega_{0}$ for some $C>0$. Then

$$
-C e^{C t} \omega_{0} \leqslant \operatorname{Ric}\left(\widetilde{\omega}_{t}\right)=\operatorname{Ric}\left(\omega_{0}\right)-\sqrt{-1} \partial \bar{\partial}\left(\frac{\partial u}{\partial t}+u\right),
$$

and we could only have

$$
C \omega_{0}-\sqrt{-1} \partial \bar{\partial}\left(e^{-C t}\left(\frac{\partial u}{\partial t}+u\right)\right) \geqslant 0 .
$$

Applying the same discussion as before for $e^{-C t}\left(\frac{\partial u}{\partial t}+u\right)$ only gives

$$
\frac{\partial u}{\partial t}+u \geqslant-C e^{C t}
$$

Hence, the metric bound corresponding to Proposition 3.1 is

$$
e^{-C e^{C t}} \omega_{0} \leqslant \widetilde{\omega}_{t} \leqslant e^{C t} \omega_{0},
$$

which is not enough to draw any decent conclusion.

Remark 3.3. With this general lower bound of Ricci curvature for infinite time singularity case, when $X$ is a projective manifold of general type, i.e., $\left(-c_{1}(X)\right)^{n}>0$, by the results in [12], one has the Ricci curvature being bounded from both sides and $\frac{\partial u}{\partial t}+u \geqslant-C$. Thus the metric bound can be improved to $C e^{-C t} \omega_{0} \leqslant \widetilde{\omega}_{t} \leqslant e^{C t} \omega_{0}$, not yet good enough. Notice that by Corollary 3.2, $D$ has to be strictly bigger than one to have a reasonable Ricci lower bound assumption for this case.

\section{References}

[1] Cao, Huaidong: Deformation of Kaehler metrics to Kaehler-Einstein metrics on compact Kaehler manifolds. Invent. Math. 81(1985), no. 2, 359-372.

[2] Hamilton, Richard S.: The formation of singularities in the Ricci flow. Surveys in differential geometry, Vol. II (Cambridge, MA, 1993), 7-136, Int. Press, Cambridge, MA, 1995.

[3] Sesum, Natasa: Curvature tensor under the Ricci flow. Amer. J. Math. 127 (2005), no. 6, 1315-1324.

[4] Song, Jian; Tian, Gang: The Kähler-Ricci flow on surfaces of positive Kodaira dimension. Invent. Math. 170 (2007), no. 3, 609-653. 
[5] Song, Jian, Weinkove, Ben: Contracting exceptional divisors by the KählerRicci flow. arXiv:1003.0718 (math.DG).

[6] Tian, Gang: On Kähler-Einstein metrics on certain K "ahler manifolds with $C_{1}(M)>0$. Invent. Math. 89 (1987), no. 2, 225-246.

[7] Tian, Gang; Zhang, Zhou: On the Kähler-Ricci flow on projective manifolds of general type. Chinese Annals of Mathematics-Series B, Volume 27, a special issue for S. S. Chern, Number 2, 179-192.

[8] Tsuji, Hajime: Existence and degeneration of Kaehler-Einstein metrics on minimal algebraic varieties of general type. Math. Ann. 281(1988), no. 1, $123-133$.

[9] Wang, Bing: On the conditions to extend Ricci flow. Int. Math. Res. Not. IMRN 2008, no. 8, Art. ID rnn012, 30 pp.

[10] Zhang, Zhou: General weak limit for Kahler-Ricci flow. arXiv:1104.2961 (math.DG).

[11] Zhang, Zhou: Scalar curvature behavior for finite-time singularity of Kähler-Ricci flow. Michigan Math. J. 59 (2010), no. 2, 419-433.

[12] Zhang, Zhou: Scalar curvature bound for Kähler-Ricci flowa over minimal manifolds of general type. Int. Math. Res. Not. IMRN 2009, no. 20, 39013912.

[13] Zhang, Zhou: Degenerate Monge-Ampere equations over projective manifolds. Thesis (Ph.D.)-Massachusetts Institute of Technology. 2006. (no paging), ProQuest LLC, Thesis.

Email: zhangou@maths.usyd.edu.au 